\section{CONTRIBUTIONS TO MOLECULAR PHYSICS IN HIGH VACUA ${ }^{1}$}

THIS paper is a continuation of the Bakerian Lecture "On the Illumination of Lines of Molecular Pressure and the Trajectory of Molecules," read before the Royal Society, December 5, 1878. Phenomena there briefly referred to have since been more fully examincd; new facts have been observed, and their theoretical bearings discussed ; and numerous experiments suggested by Prof. Stokes and others have been tried, with the result of acquiring much information which cannot fail to be of value in assisting to evolve a theory capable of embracing all the phenomena under discussion.

Experiments previously described have shown that the molecular stream hypothesis is the correct one. According to this, the molccules of the residual gas, coming in contact with the negative pole, acquire a negative charge, and inmediately fly off by reason of the mutual repulsion exerted by similarly electrified bodies. Were the individual molecules solely acted on by the initial impulse from the negative pole, they would take a dircction accurately normal to the surface repelling them, and would start with their full velocity. But the molecules, being all negatively electrified, exert mutual repulsion, and therefore diverge laterally. The negative pole, likewise, not only gives an initial impulse to the molecules, but it also continues to act on them by repulsion, the result being that the molecules move with an accelerating velocity the further they get from the polc. The lateral divergence of the molecules, owing to their negative electricity, will naturally increase with the amount of charge they carry; the greater the number of collisions the more the molecules lose negative charge, and the less divergent the stream becomes. This hypothesis is botnc out by facts. When the vacuum is just good enough to allow the shadow to be seen, it is very faint (owing to few molecular rays), but is quite sharp (owing to the divergence of the molecules laterally). The variation in mutual repulsion is shown by the fact that the focus projected from a concave pole falls beyond the centre of curvature, and varies in position with the exhaustion, being longer at high than at low exhaustions.

Assuming that the phosphorcscence is due, either directly or indirectly, to the impast of the molecules on the phosphorescent surface, it is reasonable to suppose that a certain velocity is required to produce the effect. Within the dark space, at a moderate exhaustion, the velocity does not accumulate to a sufficient extent to produce phosphorescence; but at higher exhaustions the mean frec path is long enough to allow the molecules to get up speed sufficient to cause phosphorescence. At a very high cxhaustion the phosphorescence takes place nearer the negative pole than at lower exhaustions; this I consider results from the initial velocity of the molecules bcing sufficient to produce phosphorescence, their greater speed being due to the fewer collisions near the negative pole.

The luminous boundary to the dark space round the negative pole is probably due to the impact of molecule against molccule, producing phosphorescence of the gas in the same way as the impact of molecules against German glass produces phosphorescence of the glass.

The following experiments were commenced at the suggestion of Prof. Maxwell :-

$\Lambda$ tube was made as sbown in Fig. I. The terminal $a$

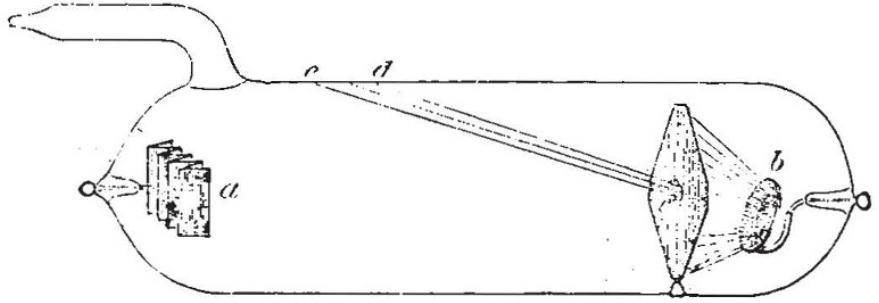

F1G. x.

is a rectangular plate of aluminium, folded as shown in section Fig. 2 ; the other terminal $b$ is a flat disk of aluminium set obliquely to the axis of the tube. In front of the pole $b$ is fixed a screen of mica, with a smail ho!e in it, as shown at $c$; this hole is not in the axis of the tube, but a little to one side of it, so that rays starting normally from the centre of the pole $b$ may pass through it and strike the glass at $d$, whilst at the same time rays passing direct between the poles $a$ and $b$ can also pass through the holc.

The questions which this apparatus was to answer are : (I) Will there be molecular projections from the negative pole, $a$, in two scries of plane strata normal to the sides of the individual furrows, or will the projection be perpendicular to the clectrode as a whole, i.e., along the axis of the tube? and (2), Will the molecular rays from the pole $b$, when it is made negative, issue throtgh the aperture of the screen, along the axis of the tube, i.e., direct to the positive pole, or will they leave the pole normal to its surface and strike the glass as shown at $d$ ?

The tube was exhausted and connected with an induction coil ; the following results were obtained: $-A$ t a moderate exhaustion, the corrugated pole being made

1 "Contributions to Molecular Physics in High Vacua. Mannctic Detlection of Mrolecular Trijecinry; Laws of Magnetic Rotation in "High and I.now Vacua; Phosphorozente P'roperties of Molccular Discharge." By William Cruokes, F.R.S. (Extracts from a paper in the L'sios phlitial Iransactions of the R yal Society, Part 2, iS79)

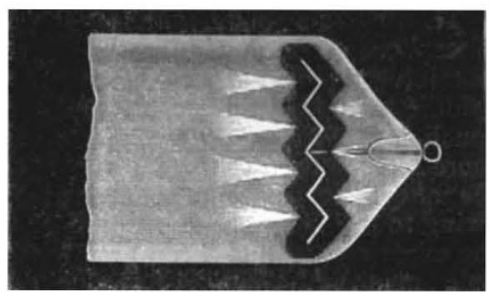

FIG. 2.

negative, the dark space entircly surrounds it, slight indentations being visible opposite each hollow, where there also is a linear concentration of blue light. The appearance is in section as shown in Firr. 2. At higher exhaustions the luminous margin disappears and the rays which previously formed the blue foci are now projected on the inner surface of the tulse, where they make themsclves evident in reen phosphorescent light as portions of ellipses formed by the intersection of the several sheets

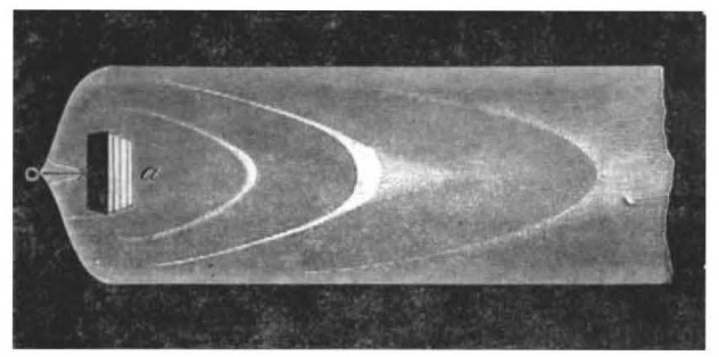

FIci. 3.

of molecular rays with t':e cylin irical tube. Fig. 3 shows this appearance.

When the other po!e was made negative, and the 
exhaustion was such that the dark space extended about 8 millims. from the pole, the first appearance noticed was that of a ray of dark blue light issuing through the hole in the mica screen, and shooting upwards towards the side of the tube, but not reaching it. Fig. 4 shows the

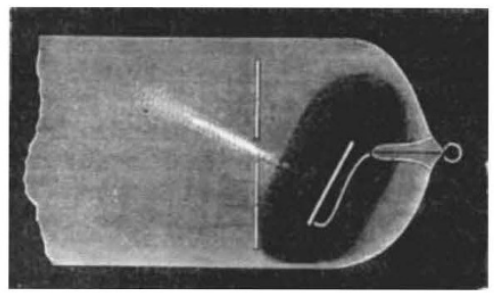

FIG: 4.

dark space round the pole, and the ray of blue light. On increasing the exhaustion this blue line of light, and the luminous boundary to the dark space, disappeared, and presently a green oval spot appeared on the side of the tube, exactly on the place previously marked where the rays issuing normal from the surface of the pole should fall.

It happened that this oval spot fell on a portion of the tube where one of the elliptical projections from the opposite (corrugated) pole also fell when that was made negative. Thus by reversing the commutator I could get a narrow band of green phosphorescent light from one pole, or a wider oval of green light from the other pole, to fall alternately on the same portion of the glass. Fig. 5 shows these effects, which, however, did not occur together as represented in the figure, but alternately.

The narrow band shone very brightly with green phosphorescence, but on reversing the commutator and obtaining the oval spot, this was seen to be cut across the middle by a darker band where the phosphorescence was much less intense. The light of the band was always more intense than that from the spot; the impacts from the one being more concentrated than from the other, owing to the shape and position of the poles; moreover the experiments had been first tried with the corrugated pole negative. The glass along the band gradually becomes deadened by repeated impacts, and will not readily phosphoresce in reply to the weaker blows from the flat plate, although it still responds to the more energetic bombardment from the corrugated pole. This phenomenon almost disappears at very high exhaustions, or if the tube is allowed to rest for some time. The tired glass then recovers its phosphorescent power to some extent, but not completely.

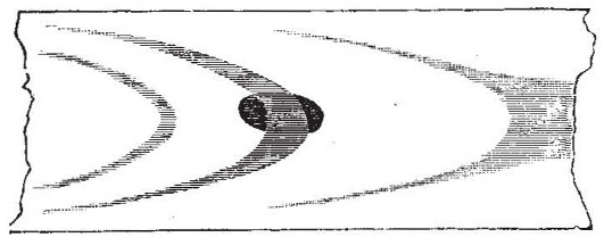

FIG. 5

To obtain this action in a more striking manner, a tube was made having a metal cross on a hinge opposite the negative pole. The sharp image of the cross was projected on the phosphorescent end of the bulb, where it appeared black on a green ground. After the coil had been playing for some time a sudden blow caused the cross to fall down, when immediately there appeared on the glass a bright green cross on a darker background. The part of the glass formerly occupied by the shadow, having been protected from bombardment, now shone out with full intensity, whilst the adjacent parts of the glass had lost some of their sensitiveness, owing to previous bombardment.

This effect of deadening produced on glass by longcontinued phosphorescence was shown in a very striking manner at a lecture delivered at the Royal Institution on April 4, 1879, when the image of a cross was stencilled on the end of a large pear-shaped bulb.

I subsequently experimented further with this bulb, and found that the image of the cross remained firmly stencilled on the glass. The bulb was then opened and the wide end heated in the blowpipe flame till it was quite soft and melted out of shape. It was then blown out again into its original shape, and re-exhausted; on connecting it with the induction coil, the metal cross being down out of the line of discharge, the original ghost of the cross was seen to be still there, showing that the deadening of the phosphorescing powers the glass produced by the first experiment at the Royal Institution had survived the melting-up and re-blowing out of the bulb.

When experimenting with this apparatus a shifting of the line of molecular discharge was noticed when the current was first turned on. The flat pole $b$ (Fig. 6) being

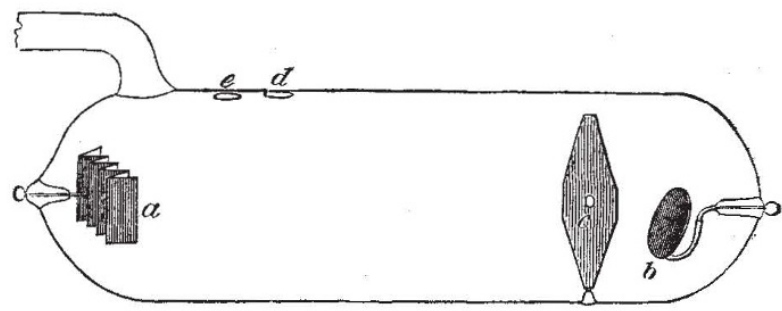

Fig. 6 .

negative and the line $c d$ being normal to its surface, the spot of light falls accurately on $d$, when the exhaustion is sufficiently good to give a sharp oval image of the hole $c$. But at higher exhaustions, when the outline of the image of $c$ becomes irregular and continually changing, the patch of light at the moment of making contact is sometimes seen at $e$, and then almost instantly travels from $e$ to $d$, where it remains as long as the current passes. The passage of the spot from $e$ to $d$ is very rapid, and requires close attention to observe it. If the coil is now stopped for a longer or shorter time, and contact is again made the same way as before ( $b$ being negative), the spot does not now start from position $e$, but falls on $d$, in the first instance. This can be repeated any number of times.

If now the pole $b$ be made positive even for the shortest possible interval, and it then be made negative, the original phenomenon occurs, and the spot of light starts from $e$ and rapidly travels to $d$. After this it again falls on $d$, $a b$ initio, each time contact is made, so long as $b$ is kept the negative pole. There seems no limit to the number of times these experiments can be repeated. The explanation of this result appears to depend on a temporary change in the condition of the wall of the glass tube when positively electrified molecules beat against it, a change which is undone by subsequent impact from negative molecules. This phenomenon is closely connected with some shadow and penumbra experiments described further on, and as the same explanation will apply to both I will defer any theoretical remarks for the present.

A suggestion was made by Prof. Maxwell that I should introduce a third, idle, electrode in a tube between the positive and negative electrodes so that the molecular stream might beat upon it, so as to see if the molecules gave up any electrical charge when impinging on an obstacle. A tube was therefore made as shown in Fig. 7; $a$ and $b$ are the ordinary terminals; $c$ and $d$ are large aluminium disks nearly the diameter of the tube, con- 
nected with outer terminals. The poles $a$ and $b$ were connected with the induction coil, an earth wire was brought near the idle pole $c$, and a gold leaf electroscope was brought near $d$.

On passing the current at inferior exbaustions, when the dark space is about 8 millims. from the negative pole,

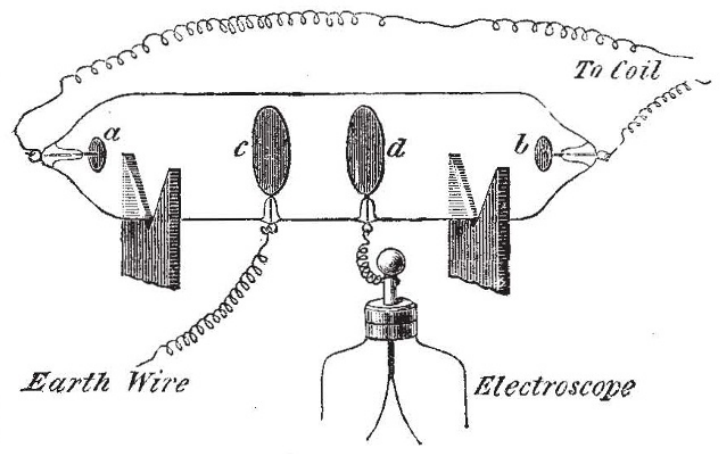

Fig. 7.

no movement of the gold leaves takes place whether $a$ or $b$ is negative, and whether $c$ is connected with earth or is insulated.

At a good exhaustion, when the green phosphorescence of the glass is strong, the gold leaves are only slightly affected whichever way the current passes.

On increasing the exhaustion to a very high point, so that the green phosphorescence gets weaker and the spark has a difficulty in passing, the gold leaves are violently affected. When the pole $a$ is negative and $b$ positive, the leaves diverge to their fullest extent. On examining their potential it is found to be positive. The coil was stopped and the gold leaves remained open. A touch with the finger caused them to collapse. They then gradually opened again, but not to the original extent. The finger again discharged them, when they reopened slightly a third time. Experiment showed that the electrical excitement took many minutes to recover equilibrium. A Leyden jar put to the idle pole $d$ was charged positively.

The earth wire and electroscope remaining, as shown in the figure, the direction of current was reversed so as to make $a$ positive and $b$ negative. The gold leaves were now less strongly affected; they opened a little, and remained quivering, as if under the influence of rapidlyalternating currents.

The wires were rearranged as shown in Fig. 8, $b$ and $d$

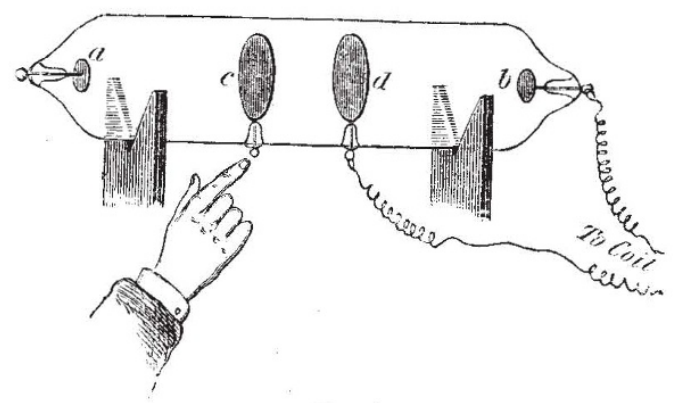

FIG. 8.

being connected with the coil. When $d$ was made negative, faint sparks about I millim. Iong could be drawn by the finger from $c$; but when $d$ was made positive the sparks from $c$ were to millims. long. The same results are obtained when the finger is brought near $a$, so long as $c$ remains insulated. If, however, $c$ be connected with earth by a wire, no sparks can be got from $a$, whichever way the current passes between $b$ and $d$. Connecting $a$ with earth diminishes the length of the sparks, which can be drawn from $c$ by about one-half.

The poles $a$ and $b$ being connected with the coil and the idle poles $c$ and $d$ having loose wires hanging from them, the wires were strongly repelled from each other.

The above experiments show that an idle pole in the direct line between the positive and the negative poles, and consequently receiving the full impact of the molecules driven from the negative pole, has a strong positive charge.

It now became of interest to ascertain whether the trajectory of the molecules suffered any deflection in passing an idle pole when it was suddenly uninsulated by an earth contact. For this purpose I used the tube described in a former paper, ${ }^{\mathrm{T}}$ where the shadow of an aluminium star was projected on a plate of phosphorescent glass. So long as the aluminium star is insulated, the shadow is sharp, as already described; but on touching the star to earth, the shadow widens out, forming a tolerably welldefined penumbra outside the original shadow, which can still be seen unchanged in size and intensity. On removing the earth connection, the penumbra disappears, the umbra remaining as before. The same penumbra is produced by connecting the idle pole with the negative pole through a very high resistance, such as a piece of wet string, instead of connecting it with earth. On bringing a magnet near the negative pole, the shadow of the (insulated) star is much increased in definition, the adjacent luminous parts of the screen becoming more luminous. Touching the star now brings a large, somewhat blurred, penumbra round the original image. The penumbra obeys the magnet the same as the umbra.

The aluminium star was now made the positive pole, the other pole remaining unchanged. The shadow of the star was projected on the phosphorescent plate of the same sharpness and almost the same intensity of light and skade as if the positive pole had been the one ordinarily used as such. The image obeyed the magnet as usual. With this arrangement the penumbral action could not be tested.

This, therefore, confirms the above-described resultsthat the idle pole, the shadow of which is cast by the negative pole, has strong positive charge. Now the stream of molecules must be assumed to carry negative electricity; when they actually strike the idle pole they are arrested, but those which graze the edge are attracted inwards by the positive electricity, and form the shadow. When the idle pole is connected with earth its potential would become zero were the discharge to cease; but, inasmuch as a constant positive charge is kept up from the passage of the current through the tube, we must assume that the potential of the uninsulated idle pole is still sufficiently positive to neutralise the negative charge which the impinging molecules would give it, and leave some surplus of positive. The effect of alternately uninsulating and insulating the idle pole is therefore to vary its positive electricity between considerable limits, and consequently its attractive action on the molecules which graze its edge. ${ }^{2}$

Experiments were tried with an idle pole and shadow tube whilst the exhaustion was going on. At such a rarefaction that the shadow can ju,t be made out, it is quite sharp; touching the idle pole causes a small penumbra to appear round its shadow. When the exhaustion is at the best point for obtaining the green phosphorescence on the glass, the shadow is very sharp and well defined; and connecting the idle pole with earth gives a much wider penumbra, the width of the penumbra increasing with the degree of rarefaction. When the

$x$ Phil. Trans., r8ิ79, vol. 170, p. 147.

2 I am aware that the theory which makes these effects of deflection de pend on electrostatic attractions and repulsions is open to some grave objections: still it was that which in a great measure guided me in my experiments, and it could not well be omitted without reducing the description of them to a dry record of apparently unconnected facts. 
vacuum is so high that the spark has difficulty in passing, the penumbra (which becomes visible on insulating the idle pole) is mu:h wider than before, and apparently eight or ten times as wide as it was at the lowest exhaustion at which obscrvations were taken.

If the object whosc shadow is cast on the screen is a non-conductor (such as a piece of glass rod), its shadow remains constant at all exhaustions, no penumbra beingr visible, as it cannot be uninsulated.

Prof. Stokes, whose suggestions throughout the course of this research have been most valuable, considered that much information might be gained by experimenting with an apparatus constructed in the following manner: the tivo poles of the tube (Fig. 9) are at $a$ and $b$. At $c$ is a

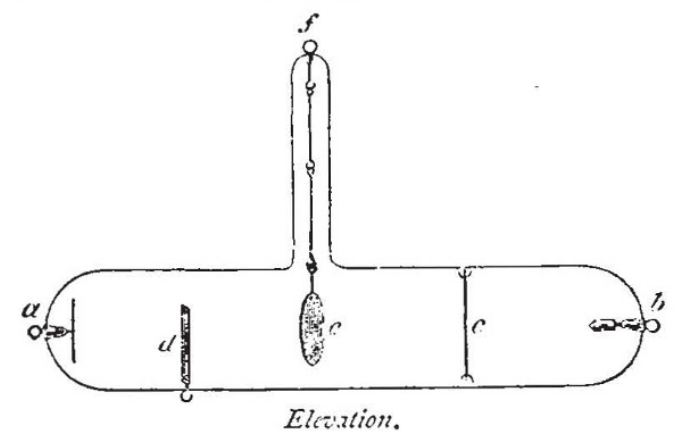

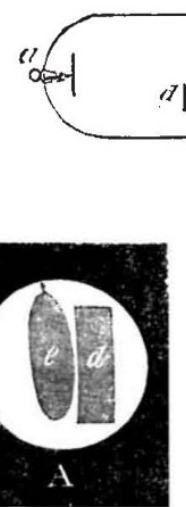

Fig. 9 A.
Elcistion.

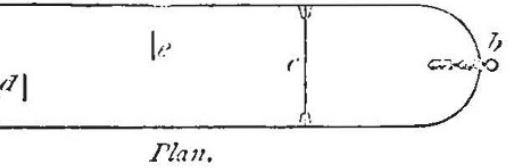

Fis. 9.

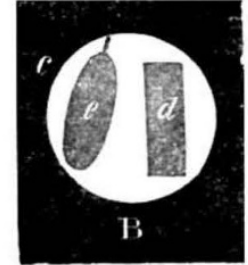

Fic. $\mathrm{g}$ i.

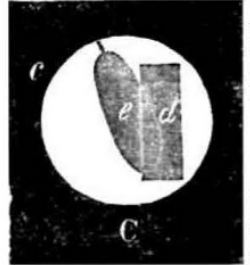

FIc. co. fluorescent screen; $d$ is a fixed bar of aluminium, and $c$ is another aluminium bar hanging from a platinum pole $f$, by a metal chain. 'The bar and pendulum are on opposite sides of the horizontal axis of the tube, as shown in the plan, so that when properly cxhausted and the pole $a$ made negative, the sbadows of bar and pendulum shall fall side by side on the screen, as shown in Fig. 9.1. On swinging the pendulum, the shadow alternately overlaps and recedes from the shadow of the bar (Figs. 9!? and $9 \mathrm{C}$ ).

This apparatus was tricd many times with an induction coil, and also with a Holtz machine; but the results were not sufficiently definite to render it safe to draw any inferencefrom them. Iy the kindness of Mr. I)e La Rue I have lately had the opportunity of experimenting with his large chloride of silver battery, and the results now come out with great sharpness and with none of the fickering and indecision met with when working with an induction-coil.

The tube was so adjusted that the pendulum huner free, and a narrow line of molectilar discharge passed between the edges of the bar and the pendulum, forming a line of light between the two shadows on the screen (Fig. 9A). When the pendulum was set swinging, and the idle pole $f$ connected with it was kept insulated, the regular appearance of the moving and fixed shadows was very slightly interfered with. That is to say, the shadows followed the successive positions between those shown in Figs. 9P? and $9 \mathrm{C}$ almost as if they had been cast by a luminous point in place of the negative pole. As the shadow of the swinging pendulum came very near that of the bar, the latter shadow scemed to shrink aray, showing that the pendulum itself excrted slight repulsion on the molecules which passed close to its edge.

The pendulum was again set stationary, as shown on the plan (Fig IO), the line of light separating the two

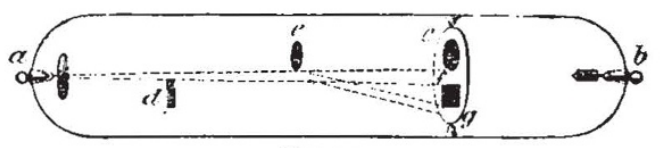

FIG. 10.

being at $f$, so that the appearance on the screen was as shown at Fig. 9A. The pendulum pole was then connected with earth, and instantly the line of light which separated the poles moved from $f$ to $g$ through an angle, measured from $e$, of about $3 \mathrm{O}^{\circ}$, the shadow widening out and getting indistinct at the same time.

When the pole $a$ was negative and $b$ positive, the bar $d$ and penclulum $c$ were each found to be positively electrified. The outside of the glass tube, both near the negative pole and near the positive pole, was also positively electrified.

The above experiments were tried with 6300 cells, a resistance equal to $\$ 00,000 \mathrm{ohms}$ being interposcd. The current through the tube was $0^{\circ} 00_{3} \mathrm{~S}_{3}$ weber. These measurements werc taken by Mr. De La Rue, to whom I am greatly indebted for permission to experiment with his magnificent battery, and who himself kindly assisted me in making the arrangements. IVHLLIA.I CROOKES

( $T$ o be continued, )

ROCK-WEATHERING, AS IILUSTRATED IN CHURCHYARDS

COMPARATIVELY little has yet been done in the way of precise measurement of the rate at which the exposed surfaces of different kinds of rock are removed in the processes of weathering. A few years ago some experiments were instituted by Prof. Pfaff of Erlangen to obtain more definite information on this subject. He exposed to ordinary atmospheric influences carefully measured and weighed pieces of Solenhofen limestone, syenite, granite (both rough and polished), and bone. At the end of three years he found that the loss from the limestone was equivalent to the removal of a uniform layer $0.04 \mathrm{~mm}$ in thickness from its general surface. 'The stone had become quitc clull and earthy, while on parts of its surface fine cracks and incipient exfoliation bad appcared. ${ }^{2}$ The time during which the observations were continued is however too bricf to allow any general deductions to be drawn from them as to the real average rate of disintegration. Prof. Pfaff relates that during the period a scvere hailstorm broke one of the plates of stonc. An exceptionally powerful cause of this nature might make the loss during a short interval considerably greater than the true average of a longer period.

It occurred to me recently that data of at least a provisional value might be obtained from an examination of tombstones freely exposed to the air in graveyards in cases where their dates remained still legible or might be otherwise ascertained. I have accordingly paid attention to the older burial-grounds in Edinburgh, and have gathered togetber some facts which have perhaps sufficient interest and novelty to be communicated to the Socicty.

At the outset it is of course obvious that in seeking for 'A Paree read to the Royal Society of Iuinburgh, on April xo, by P'rnf. Gieikie, F.R.S.

${ }_{2}$ "Allgemei:ie Geolc gie aIs exacte Wissenschaft," p. 317. 